\title{
Enol and Enethiol Occurrence for Some Ketones and Thioketones. Mass Spectrometry and Theoretical Calculations
}

\author{
Patricia E. Allegretti ${ }^{1}$, María de las Mercedes Schiavoni ${ }^{1}$, María Susana Cortizo ${ }^{2}$, Eduardo A. \\ Castro $^{3, *}$ and Jorge J. P. Furlong ${ }^{1}$ \\ ${ }^{1}$ Laboratorio de Estudio de Compuestos Orgánicos (LADECOR), División Química Orgánica, \\ Departamento de Química, Facultad de Ciencias Exactas, Universidad Nacional de La Plata, (1900) \\ La Plata, Buenos Aires, Argentina. \\ ${ }^{2}$ Instituto de Investigaciones Físico-Químicas, Teóricas y Aplicadas (INIFTA), Facultad de Ciencias \\ Exactas, Universidad Nacional de La Plata, (1900) La Plata, Buenos Aires, Argentina. \\ ${ }^{3}$ INIFTA (UNLP-CONICET), Departamento de Química, Facultad de Ciencias Exactas, Universidad \\ Nacional de La Plata, Suc.4, C.C. 16, (1900) La Plata, Buenos Aires, Argentina.
}

* Author to whom correspondence should be addressed; E-mail: castro@quimica.unlp.edu.ar, Tel. 54-(0)221-4257430, Fax: 54-(0)221-4254642

Received: 31 January 2004; in revised form: 9 October 2004 / Accepted: 5 November 2004 / Published: 30 November 2004

\begin{abstract}
The mass spectra of ketones can provide valuable information with regards to keto-enol equilibria occurring in the gas phase. Mass spectra of selected ketones and thioketones have been analysed and specific fragmentation assignments have been done to characterised and weigh co-existing keto and enol tautomers. Thioketones are of particular interest due to their tendency to shift the tautomeric equilibrium towards the enethiol form. The predictive value of this methodology is not only supported by the influence of these compound's nature and size of the substituent on these equilibria but also by the good correlation found between the selected fragments abundances ratio and semi-empirical calculation (AM1) of the corresponding heats of tautomerization.
\end{abstract}

Keywords: Tautomerism, ketones, mass spectrometry, theoretical calculations 


\section{Introduction}

The understanding of tautomerism and its corresponding equilibrium nature is relevant to the study of many molecular processes in Organic Chemistry and Biochemistry [1,2]. Although enols are thermodynamically unstable species in comparison with their carbonyl isomers, some of them have a long enough lifetime so as to be detected by conventional methods. They are dramatically stabilized by the introduction of bulky groups in the carbon $\alpha$ - to the carbonyl group [3-5] and, in some cases, the enol form can be the thermodynamically most stable $[6,7]$.<smiles>[R]C([R])C([R])[R]</smiles>

The thione group is relatively unstable in the monomeric form and tends to turn into a stable C-S single bond [8]. As already established in previous work, the heteroatom O-S exchange exerts a noticeable effect on the equilibrium position [9]. Thus, aliphatic thioketones exist in equilibrium with their corresponding enethiols [10].

From theoretical calculations, the keto and thioketo structures of simple carbonyl compounds are 11 and $4 \mathrm{kcal} \mathrm{mol}^{-1}$ more stable than the enol and enethiol structures, respectively. We conclude that $\mathrm{pK}$ thioenol values should be lower than the corresponding $\mathrm{pK}$ enol values [11]. In fact, tautomerism of organic compounds has been subject of theoretical studies by using several quantum-mechanical approaches $[5,12,13]$.

As the thiocarbonyl group exhibits characteristic absorption in the UV/VIS region, the thione form can be detected spectrophotometrically [14]. The prototropic tautomerism of thiones can also be detected by infrared spectrometry [15] and polarography [16]. The thiol form of 2(1H)-pyridine thione was predicted to be predominant in the gas phase, not only by experimental methods (IR) but also by theoretical calculations [17]. Definite and quantitative data for keto-enol equilibria are available by nuclear magnetic resonance (NMR) spectrometry [18-20]. As described in the literatura, during the last few years, the same results were obtained in the thione series, in which the tautomers seem to be relatively slowly interconverted and therefore the proton signals of the tautomeric isomers may be expected to be clearly distinguishable $[21,22]$. In polar solvents both tautomeric forms are detectable by both UV and NMR spectrometries [23].

Cyclic thioketones and cyclic $\beta$-dithioketones were examined by mass spectrometry [24]. Compared with the oxygen analogues, the thioketones yielded a higher proportion of molecular ions, indicating the greater ability of the sulfur atom to stabilise the radical cation. The loss of SH was found to be a characteristic fragmentation. The results obtained by mass spectrometry when correlated with those from molecular orbitals for amides, ureas, hydantoins, isoquinolinones, ketones, diketones, lactones, etc. demonstrate that mass spectrometry constitutes an adequate tool for predicting tautomeric equilibrium shifts within a family of compounds $[5,12,25]$.

This work deals with the study of keto-enol equilibria for some ketones and thio analogues by resorting to mass spectrometry and semi-empirical calculations. 


\section{Results and Discussion}

\section{Gas Chromatography-Mass Spectrometry}

Table 1 shows the most relevant mass spectral data for selected ketones. To estimate the occurrence of both tautomers in equilibrium, appropriate fragmentations have been assigned to the keto and enol forms. The loss of $\mathrm{OH}$ or $\mathrm{H}_{2} \mathrm{O}$ from the molecular ion can be assigned to the enol form and the ${ }^{C H R}{ }^{2} \mathrm{R}^{3}$ radical loss to the keto form (except for the symmetrical ketones, where this assignment does not apply). Their abundance ratios can be an adequate indicator of the enol/keto occurrence, provided that the corresponding fragments are highly specific with regards to their assignment (i.e. free from spectral interferences). For the compounds in Table 1 this is in fact the case, meaning that there are not other fragments with the same mass but arising from different fragmentation pathways.

Table 1. Relevant Mass Spectral Data for Selected Ketones. ${ }^{\text {a }}$

\begin{tabular}{|l|c|c|c|c|c|}
\hline \multicolumn{1}{|c|}{ Compound } & $\mathbf{M}^{+.}$ & $\mathbf{( M - O H}^{+}$ & $\left.\mathbf{( M - H}_{\mathbf{2}} \mathbf{O}\right)^{+\cdot}$ & $\left.\mathbf{( M - ~} \mathbf{C H R}^{2} \mathbf{R}^{3}\right)^{+}$ & $\begin{array}{c}\left.\mathbf{1 0}^{2} \mathbf{( M - O H}\right)^{+} / \\
\mathbf{( M - C H R}^{\mathbf{2}} \mathbf{R}^{+}\end{array}$ \\
\hline 3-Methyl-2-butanone & 133 & - & - & 613 & - \\
Methylphenylketone & 95.1 & 1.5 & - & 324 & 0.5 \\
Methylnaphtylketone & 109 & 2.1 & - & 227 & 0.9 \\
Isopropylphenylketone & 27.4 & 3.4 & - & 338 & 1.0 \\
2-Methoxyphenylmethylketone & 43.8 & 6.2 & 6.5 & 296 & 2.1 \\
3-Methoxyphenylmethylketone & 149 & 2.3 & - & 251 & 0.9 \\
4-Methoxyphenylmethylketone & 135 & 0.7 & - & 371 & 0.2 \\
2,4-Dimethoxyphenylmethylketone & 139 & 12.3 & 6.0 & 286 & 4.3 \\
4-Ethylphenylmethylketone & 58.8 & 2.0 & 3.1 & 284 & 0.7 \\
Methyl-2-nitrophenylketone & 1.2 & - & - & 239 & - \\
2-Bromophenylmethylketone & 41.2 & - & - & 156 & - \\
\hline
\end{tabular}

${ }^{a}$ The reported electron impact ion abundances were calculated according to the following ratio: (10 $0^{3}$ Ion Abundance)/(Total Ion Abundance)

Despite the fact the analyses have been carried out by GC-MS, no chromatographic separation has been observed so that the mass spectra are the result of the superposition of both tautomers. Contrarily, previous work has reported chromatographic separation of the tautomeric forms for $\beta$-ketoesters [26]. From Table 1 the tendency observed for methylketones $\left(\mathrm{R}^{1}\right.$ : isopropyl, phenyl and naphtyl respectively), as previously reported [5], is easily explained in terms of the steric hindrance exerted by the substituent $\mathrm{R}^{1}$ next to the carbonyl group (Eq. 1). The increase of enol occurence for isopropylphenylketone respect to methylphenylketone can also be explained in terms of steric hindrance.

In the case of methylphenylketones an enol/keto ratio increase is observed for 2-methoxy and 2,4dimethoxyphenylmethylketone, which is not assigned to ortho steric effect, since it is not consistent with the data obtained for methyl-2-nitrophenylketone and 2-bromophenylmethylketone, ketones 
which exhibit an important steric effect, main responsible for their occurrence as keto tautomers. The role of the methoxy group as an electrodonor substituent does not seem to apply either since the substitution with an ethyl group in the para position does not exert a noticeable influence.

A reasonable explanation for 2-methoxyphenylmethylketone is the stabilization of the enol form by intramolecular hydrogen bonding which is not possible for the other methylphenylketones (Eq. 2).

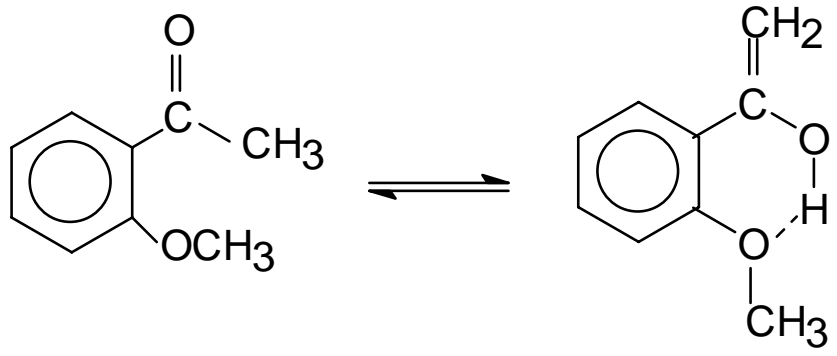

Table 2 shows the most relevant mass spectral data for selected ketones and thioketones. For propanone it should be taken into account that for such small molecules unspecific rearrangements can occur, in which case the enol/keto ratio (from the selected fragments) does not apply.

Table 2. Relevant Mass Spectral Data for Selected Ketones and Thioketones ${ }^{\mathrm{a}}$.

\begin{tabular}{|c|c|c|c|c|c|}
\hline Compound & $\mathbf{M}^{+}$ & $(\mathbf{M}-\mathrm{XH})^{+}$ & $\left(\mathbf{M}-\mathbf{X H}_{2}\right)^{+.}$ & $\left(\mathbf{M}-\mathrm{CHR}^{2} \mathbf{R}^{3}\right)^{+}$ & $\begin{array}{c}\mathbf{1 0}^{2}(\mathbf{M}-\mathrm{XH})^{+} / \\
\left(\mathbf{M}-\mathrm{CHR}^{2} \mathbf{R}^{3}\right)^{+}\end{array}$ \\
\hline Propanone & 162 & 8.5 & 3.8 & $500^{\mathrm{b}}$ & n.a. (1.7) \\
\hline Propanethione & 218 & 46.5 & 13.1 & $376^{\mathrm{b}}$ & n.a. (12.4) \\
\hline 3-Methyl-2-butanone & 133 & - & - & 613 & 0.0 \\
\hline 3-Methyl-2-butanethione & 145 & 71.5 & 10.7 & 117 & 61.1 \\
\hline 2,4-Dimethyl-3-pentanone & 42.5 & - & - & $376^{\mathrm{b}}$ & 0.0 \\
\hline 2,4-Dimethyl-3-pentanethione & 140 & 87.7 & 2.8 & $57.1^{\mathrm{b}}$ & n.a. (153.6) \\
\hline Methylphenylketone & 95.1 & 1.5 & - & 324 & 0.5 \\
\hline Methylphenylthioketone & 198 & 66.1 & 11.6 & 331 & 20.0 \\
\hline Methylnaphtylketone & 109 & 2.1 & - & 227 & 0.9 \\
\hline Methylnaphtylthioketone & 189 & 84.5 & 54.9 & 279 & 30.3 \\
\hline
\end{tabular}

a The reported electron impact ion abundances were calculated according to the following ratio: ( $10^{3}$ Ion Abundance $) /($ Total Ion Abundance)

b This fragment can be assigned to both the keto and the enol tautomer, so that the calculated ratio in the last column (in parenthesis) could be higher when substracting the enol contribution from this ion abundance (n.a. stands for "not applicable").

Analysis of the results in Table 2 reveals that while enol occurrence is not very significant for the selected oxygenated ketones but with the change in heteroatoms (O-S), a strong equilibrium shift towards the enol tautomer is observed (even for propanone). 
Comparing the mass spectral data for propanethione, 3-methyl-2-butanethione and 2,4-dimethyl-3pentanethione and, analogously, for phenylmethylthioketone and methylnaphtylthioketone, it is clear that the substituent size impacts the equilibrium position: the larger the substituent, the more noticeable the shift towards the enol form [5].

\section{Theoretical Calculations}

Table 3 shows the differences between the heats of formation of the keto and enol forms for the neutral molecules and radical cations. A tendency correlation with the mass spectral data is obtained when considering the calculations for the neutral molecules. The energy requirement to yield the enol form decreases for the corresponding thio-analogues. No correlation with the mass spectral data is observed for the radical cations.

Tabla 3. Heats of Formation Difference $\left(\mathrm{kcal} \mathrm{mol}^{-1}\right)$ for the Enol-Ketone and EnethiolThioketone Transformations by AM1 Calculations.

\begin{tabular}{|l|c|c|}
\hline Compound & Neutral Molecule & Radical Cation \\
\hline Propanone & 11.21 & -0.74 \\
Propanethione & -1.47 & -1.59 \\
3-Methyl-2-butanone & 11.86 & -1.25 \\
3-Methyl-2-butanothione & -2.76 & -1.88 \\
2,4-Dimethyl-3-pentanone & 5.13 & -30.82 \\
2,4-Dimethyl-3-pentanothione & -7.68 & -10.12 \\
Phenylmethylketone & 10.67 & -1.52 \\
Phenylmethylthioketone & -1.28 & 3.95 \\
Methylnaphthylketone & 5.13 & -5.23 \\
Methylnaphtylthioketone & -14.82 & -11.53 \\
\hline
\end{tabular}

These results constitute evidence for the feasibility of tautomerism evaluation by mass spectrometry, since it seems to adequately reflect tautomeric equilibria between neutral species with no observable influence of tautomeric equilibrium between ionic species in the gas phase, which has been claimed to occur [27]. It is important to take into consideration that reactivity of radical-ionic species can differ sensibly from that of the corresponding neutral analogues.

\section{Conclusions}

There is a remarkable tendency towards enolic structures formation when sulfur replaces oxygen in the ketones here reported. This behaviour is evidenced not only by mass spectra but also by theoretical calculations and the mutual correlation. The results derived from the application of molecular orbital theory support the predictive value of the spectrometric data and the fact that they correspond to the tautomeric equilibria between neutral species, so that subsequent ionisation in the ion source does not affect the position of those equilibria. It can be concluded that mass spectrometry constitutes an 
adequate tool to predict keto-enol equilibrium occurrence for numerous members of this and diverse compound families.

\section{Experimental}

\section{Synthesis of Thioketones}

The non-commercially available compounds were synthesized according to literature procedures [28]. The synthesis of thioketones was carried out by the following general procedure: Lawesson's reagent $(5 \mathrm{mmol})$ was added to a mixture of the ketone $(10 \mathrm{mmol})$ and toluene $(10 \mathrm{~mL})$ and refluxed overnight. Upon completion of the reaction, as determined by TLC (solvent: toluene) the reaction mixture was purified by column chromatography using toluene as eluent. The extract was concentrated and purified by recrystallization to a constant melting point for solid thioketones or by distillation for liquid thioketones.

\section{Gas Chromatography-Mass Spectrometry}

These determinations were done by injection of methanol solutions $(1 \mu \mathrm{L})$ on an HP 5890 Series II Plus chromatograph coupled to an HP 5972A mass spectrometric detector under the following conditions: Column : HP5-MS, 30m x $0.25 \mathrm{~mm}$ x $5 \mu \mathrm{m}$; Carrier gas: Helium; Injector temperature: $200^{\circ} \mathrm{C}$; Oven temperature: $80^{\circ} \mathrm{C}, 10^{\circ} \mathrm{C} / \mathrm{min}, 200^{\circ} \mathrm{C}$; Interface temperature: $300^{\circ} \mathrm{C}$; Ion source: $185^{\circ} \mathrm{C}$; The pressure in the mass spectrometer $\left(10^{-5}\right.$ torr $)$ precludes ion-molecule reactions. Electron energy: 70 $\mathrm{eV}$.

\section{Computational Procedure}

Semi-empirical AM1 Molecular Orbital calculations were performed using the standard Hyperchem package [29]. Since we resorted to heat of formation values in order to rationalize experimental findings and the AM1 technique has been specially parameterized to reproduce this sort of experimental data, we deem this choice is a sensible one for the molecular set under study.

\section{References}

1. Katritzky, A.R.; Lagowski, J.M. Adv. Heterocycl. Chem. 1963, 1, 339.

2. Elguero, J.; Marzin, C.; Katritzky, A.R.; Linda, P. The Tautomerism of Heterocycles; Academic Press: New York, 1976.

3. (a) Rappoport, Z.; Biali, S. J. Am. Chem. Soc. 1986, 106, 477; (b) Rappoport, Z.; Biali, S. J. Am. Chem. Soc. 1985, 107, 1701.

4. Miller, A.R. J. Org. Chem. 1976, 41, 3599.

5. Allegretti, P.E.; Gavernet, L.; Castro, E.A.; Furlong, J.J.P. J. Molec. Struct. (THEOCHEM) 2000, 532, 139.

6. O'Neill, P.; Hegarty, A.F. J. Chem. Soc., Chem. Commun. 1987, 744.

7. Kwiatkowski, J.S.; Zielenski, T.J.; Rein, R. Adv. Quant. Chem. 1986, $18,85$. 
8. Mayer, R. Organosulfur Chemistry; Janssen, M.J., Ed.; Wiley-Interscience: New York, London, Sydney, 1967; p. 219.

9. Bordwell, F.G.; Algrim, D.J.; Harrelson Jr., J.A. J. Am. Chem. Soc. 1988, 110, 5903.

10. Dunn, F. Comprehensive Organic Chemistry, Burton, D.; Ollis, W.D., Eds.; Pergamon: New York, 1979; p. 373.

11. Sklenak, S.; Apeloig, Y.; Rappoport, Z. J. Chem. Soc., Perkin Trans. 2, 2000, 2269.

12. (a) Allegretti, P.E.; Labadie, G.R.; González Sierra, M.; Furlong, J.J.P. Afinidad 2000, VII, 485, 41; (b) Allegretti, P.E.; Gavernet, L.; Castro, E.A.; Furlong, J.J.P. Asian J. Spectrosc. 2001, 5, 63; (c) Allegretti, P.E.; Castro, E.A.; Furlong, J.J.P. J. Mol. Struct. (THEOCHEM) 2000, 499, 121; (d) Allegretti, P.E.; Milazzo, C.B.; Castro, E.A.; Furlong, J.J.P. J. Mol. Struct. (THEOCHEM) 2002, 589-590, 161.

13. (a) O'Neill, P.; Hegarty, A.F. J. Chem. Soc., Chem. Commun. 1987, 477; (b) Mukhopadhyaya, J.K.; Sklenák, S.; Rappoport, Z. J. Am. Chem. Soc. 2000, 122, 1325.

14. Fabian, J.; Mayer, R. Spectrochim. Acta 1964, 20, 299.

15. Mayer, R.; Morgenstern, J.; Fabian, J. Angew. Chem. 1964, 76, 157.

16. Reyes, Z.; Silverstein, R.M. J. Am. Chem. Soc. 1958, 80, 6367.

17. Nowak, M.J.; Lapinski, L.; Rostkowska, H.; Les, A.; Adamowicz, L. J. Phys. Chem. 1990, 94, 7406.

18. Duus, F.; Lawesson, S.O. Ark. Kem. 1968, 29, 127.

19. (a) Demuynck, C.; Demuynck, M.; Paquer, D.; Vialle, J. Bull. Soc. Chim. France 1969, 3327; (b) ibid. 3595.

20. Duus, F.; Jacobsen, P.; Lawesson, S.O. Tetrahedron 1968, 24, 5323.

21. Demuynck, C.; Demuynck, M.; Paquer, D.; Vialle, J. Bull. Soc. Chim. France 1966, 3366.

22. Demuynck, C.; Viale, J. Bull. Soc. Chim. France 1967, 2748.

23. Mayer, R.; Scheithauer, S.; Bleisch, S.; Kunz, D.; Bahr, G.; Radeglia, R. J. Prakt. Chem. 1969, 311, 472.

24. Paquer, D.; Morin, L.; Vazeux, M.; Andrieu, C.G. J. R. Chem. Soc. 1981, 100, 252.

25. (a) Allegretti, P.E.; Cánepa, A.S.; Bravo, R.D.; Castro, E.A.; Furlong, J.J.P. Asian J. Spectrosc. 2000, 4, 133; (b) Allegretti, P.E.; Peroncini, V.; Castro, E.A.; Furlong, J.J.P. Int. J. Chem. Sci. 2003, 1, 1; (c) Allegretti, P.E.; Cortizo, M.S.; Guzmán, C.; Castro, E.A.; Furlong, J.J.P. Arkivoc 2003, Part XV, 134.

26. Allegretti, P.E.; Schiavoni, M. M.; Di Loreto, H.E.; Furlong, J.J.P.; Della Védova, C. J. Molec. Struct. 2001, 560, 327.

27. (a) Budzikiewicz, H.; Djerassi, C. Chem. \& Ind. (London) 1965, 1967; (b) Larsson, F.C.V.; Lawesson, S.O.; Moller, J.; Scroll, G. Acta Chem. Scand. 1973, 27, 747.

28. Cava, M.P.; Levingston, M.I. Tetrahedron 1985, 41, 5061.

29. Hyperchem Publication HC50-01-01-00, 1996; Hypercube, Inc., Toronto, Canada.

(C) 2004 by MDPI (http://www.mdpi.org). 